\title{
SPATIAL SMOOTHING FOR ARRAYS WITH ARBITRARY GEOMETRY*
}

\author{
H. Wang ${ }^{1}, K . J . R$. Liu $^{1}$ and H. Anderson ${ }^{2}$ \\ ${ }^{1}$ Electrical Engineering Department and Institute for Systems Research \\ University of Maryland, College Park, MD 20742 \\ ${ }^{2}$ Watkins Johnson Company, Gaithersburg, MD
}

\section{ABSTRACT}

The Spatial Smoothing(S.S.) technique has been proved to be effective in decorrelating coherent signals thus making MUSIC algorithm operative in a coherent interference environment. However, such scheme can only be applied to uniformly spaced linear arrays which are known to be very sensitive to the directions of arrivals(DOAs) and can be used to estimate azimuth angles only. To significantly improve the robustness of DOA estimation, to estimate both azimuth and elevation angles, we developed techniques for applying S.S. on arrays with arbitrary geometry. We also study the cause of ambiguities in a multiple signal environment and find the necessary and sufficient conditions of an ambiguity free three-sensor array manifold. We evaluated the Forward/ Backward Spatial Smoothing on a nonlinear array with central symmetry and found that this technique outperform regular S.S. in terms of improved efficiency and estimation resolution. Finally, we expand the application of our technique to ESPRIT. All the predicted results are verified by simulations.

\section{INTRODUCTION}

Sensor array processing has been a key technology in radar/sonar, cellular communications and underwater acoustics. Much of the recent work in array processing has focused on methods for high-resolution DOA estimation. These include well known MUSIC [1] algorithm and ESPRIT [2] algorithm. However, an important drawback of these techniques is the severe degradation of the estimation accuracy in the presense of highly correlated or coherent signals. To counter the deleterious effects due to some coherent signals, a pre-processing scheme, the S.S. proposed by Evans et al. [3] and further developed by Shan et al. [4], has been shown to be an effective approach in decorrelating coherent signals. However, such scheme can only be applied to uniformly spaced linear arrays. Linear arrays are known to be limited only to DOA within $180^{\circ}$. We will also show in this paper that the accuracy of DOA estimation by performing S.S. on a linear array is very sensitive to the DOAs. As a result, a linear array is

*This work was supported in part by the NSF grant MIP-9309-506, the ONR grant N00014-93-1-0566, and MIPS/Watkins Johnson. not practically applicable in radar/sonar, and is more so in cellular communications where users can never predict the coming directions of the moving targets.

The objectives of this work are to develop S.S. techniques for an array of arbitrary geometry to gain robustness in DOA estimation and to estimate both azimuth and elevation angles. We discovered and proved the conditions on arrays with arbitrary geometry for applying S.S. These conditions are (1) such an array must have an orientation invariant structure; (2) its center array and all the subarrays must have ambiguity free array manifold; and (3) the number of subarrays is larger than or equal to the largest number of mutually coherent signals. We also proved the necessary and sufficient conditions for an ambiguity free three-sensor array manifold and then identify several situations for a higher order sensor array manifold in which ambiguity may arise, so that we can design ambiguity free center arrays and subarrays. We confirmed by simulation that S.S. can be applied to an array satisfying the aforementioned conditions (and conditions for applying $\mathrm{MU}$ SIC) to make MUSIC operative in a coherent interference environment and thus achieving robustness. To further increase efficiency and estimation resolution, we evaluated the FBSS [5], which was previously used in linear arrays, for applications in nonlinear arrays. We found this technique, when applied to a nonlinear array with central symmetry, can reduce the number of sensors and improve the estimation resolution for closely spaced incoming signals. Finally, we expand the application of our technique to ESPRIT. All the predicted performance are confirmed by simulations.

\section{ARRAY OF ARBITRARY GEOMETRY}

Consider an array of $p$ sensors. Let $d$ narrow-band signals inpinge on the array at incident angles $\theta_{1}, \ldots, \theta_{d}$ with additive White Gaussian noise. The array output covariance matrix has the form:

$$
R=E\left(r(t) r^{H}(t)\right)=A R_{s} A^{H}+\sigma^{2} I .
$$

The MUSIC algorithm is operative only under the following two conditions: (1) The matrix $A$ is of full collumn rank, i.e. the array has ambiguity free array manifold, and (2) The signal covariance matrix $R_{s}$ is full rank. 
2.1. Ambiguity Free Array Structure

The ambiguity arises when one steering vector can be expressed as a linear combination of other steering vectors in an array manifold $\mathcal{A}$. For a linear array, the rank-1 ambiguity [1] cannot be avoided since the DOAs' which are "mirror images" with respect to the array line, have the same steering vector. This limits the range of DOAs estimable by a linear array to within $180^{\circ}$. Generally, to avoid ambiguity, an array used for DOA estimation must have a proper structure. An ambiguity free array manifold has been assumed in several papers [6]- [8]. Our attempt is to identify all the situations in which ambiguity may arise.

Theorem 1 In an azimuth only system, a necessary and sufficient condition for an ambiguity free three-sensor array manifold is all these three sensors are not on one line and have mutual distance less than $\frac{\lambda}{2}$.

It is our conjecture, that a $p$ sensor array has an ambiguity free array manifold if it satisfies that no more than $\left\{\frac{p}{2}\right\}$ sensors are on the same line and for any sensor in the array, there are at least two other sensors within the range of $\frac{\lambda}{2}$. The rationale is that it is less likely to have ambiguity in higher order arrays [6].

2.2. Conditions for Applying Spatial Smoothing on Arrays with Arbitrary Geometry

When some of the incoming signals are coherent ones, the signal covariance matrix $R_{s}$ is no longer full rank. We first provide the following lemmas to obtain the necessary and sufficient conditions for applying S.S: on an array with arbitrary geometry, so that we can achieve the nonsingularity of the modified signal covariance matrix.

Lemma 1 For steering matrices $A$ and $B$, given by $A=$ $\left[a\left(\theta_{1}\right), \cdots, a\left(\theta_{d}\right)\right]$ and $B=\left[b\left(\theta_{1}\right), \cdots, b\left(\theta_{d}\right)\right]$ there exists a mapping relation $B=A C$ if and only if $C$ is an diagonal matrix given by $C=\operatorname{diag}\left\{c_{11}\left(\theta_{1}\right), c_{22}\left(\theta_{2}\right), \cdots, c_{d d}\left(\theta_{d}\right)\right\}$ with $b\left(\theta_{1}\right)=c_{11}\left(\theta_{1}\right) a\left(\theta_{1}\right), \cdots, b\left(\theta_{d}\right)=c_{d d}\left(\theta_{d}\right) a\left(\theta_{d}\right)$.

Lemma 2 For $K$ steering matrix $A_{1}, A_{2}, \cdots, A_{K}$, each $A_{i}$ can be mapped to a steering matrix $B$, if and only if there exists a mapping relation: $A_{j}=A_{i} C_{i j}$ between any $A_{i}$ and $A_{j}$.

Lemma 3 Suppose $A_{i}$ and $A_{j}$ are steering matrices associated with the ith and the $j$ th subarrays. There exists a mapping relation $A_{j}=A_{i} C_{i}$ if and only if the ith and the $j$ th subarrays are identical and have the same orientation.

From Lemma 1 to Lemma 3, we have:

Theorem 2 Suppose an array can be divided into $K$ sub. arrays, each having $a p \times d$ steering matrix $A_{;} \quad(i=$ $1,2, \cdots, K)$, then $A_{1}, A_{2}, \cdots, A_{K}$ can be mapped to a $p \times d$ steering matrix $B$ by $A_{i}=B D_{i}$ if and only if all these subarrays are identical and have the same orientation.

Definition 1 (Orientational Invariant Structure) An array has an Orientational Invariant Structure if it can be divided into $K$ subarrays and all these subarrays are identical and have the same orientation.
Definition 2 (Center Array) If an array with orientational invariant structure can be divided into $K$ subarrays, which can also be overlapped, then the collection of all the first sensors of the $K$ subarrays form a center array.

We can then get the necessary and sufficient conditions:

Theorem 3 S.S. can be applied to an array with arbitrary geometry to get a full rank smoothed signal covariance matrix if and only if an array has orientational invariant structure, its center array has ambiguity free structure, the number of subarrays $K$ is larger than or equal to the size of the largest group of coherent signals.

Based on the smoothed data covariance matrix obtained by performing S.S., MUSIC algorithm can be successfully applied.

\subsection{Further Improvement}

The FBSS technique can always be applied to a linear array to reduce the required number of sensors and to improve estimation resolution. FBSS is performed to get $\mathrm{K}$ additional backward subarrays by reversing the order of the subarrays and the order of the sensors within each subarray. For arrays of arbitrary geometry, there is some requirements on the geometry for successful implementation of the backward method. We first give the following definition of central symmetry which is a feature we identified to be necessary for applying FBSS:

Definition 3 (Central Symmetry) The array is central symmetric if it is identical before and after rotating $180^{\circ}$ about its center of mass.

We proved that generally, FBSS can be applied to an array that satisfies conditions for applying S.S. and is also central symmetric.

We found that the results obtained for a nonlinear array in the azimuth-only system remain valid in an azimuthelevation system, if all the elevations are at one side of a plane array.

\section{SPATIAL SMOOTHING FOR ESPRIT}

Similar to MUSIC, the ESPRIT algorithm [2] is another approach to the signal parameter estimation problem. It exploits an underlying data model at a significant computational savings. The ESPRIT algorithm is also limited to estimating the parameters in noncoherent incoming signals.

We found that our scheme also work for the ESPRIT algorithm to estimate parameters in the coherent interference environment. We consider each doublet sensor pair in an array with $m$ sensors used by ESPRIT algorithm as one element. Then the array consists of $\frac{m}{2}$ elements. If this array satisfies the conditions in Theorem 3 , we can get a full rank smoothed signal covariance matrix. We can then successfully perform ESPRIT.

Although the S.S. enables ESPRIT to estimate DOAs in a coherent interference environment, the estimation is still limited to identifying DOAs of signals within $180^{\circ}$ in 
an azimuth only system. Hence, in terms of performance robustness to DOA, our S.S. is more effective for MUSIC then for ESPRIT

\section{EXPERIMENTAL RESULTS}

In the first example, we use a nine-sensor linear array and a nine-sensor square array as shown in Fig.1, both witl a spacing of $0.45 \lambda$ between neighboring sensors. We consider two narrowband coherent signals with DOA at $70^{\circ}$ and $85^{\circ}$. The SNR is $20 \mathrm{~dB}$. We applied the S.S. first and then applied the MUSIC for both arrays. Figure 2. shows that the DOAs of the two coherent signals were not re solved using a linear array but a square array can give a satisfactory result.

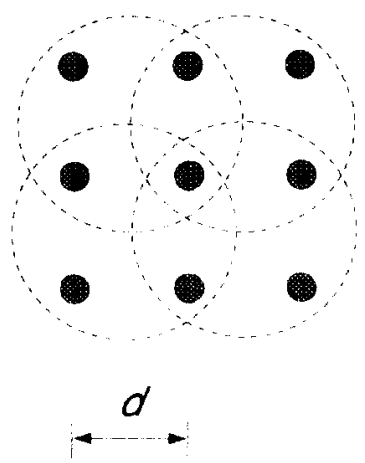

Figure 1. A nine-sensor square array with spacing d

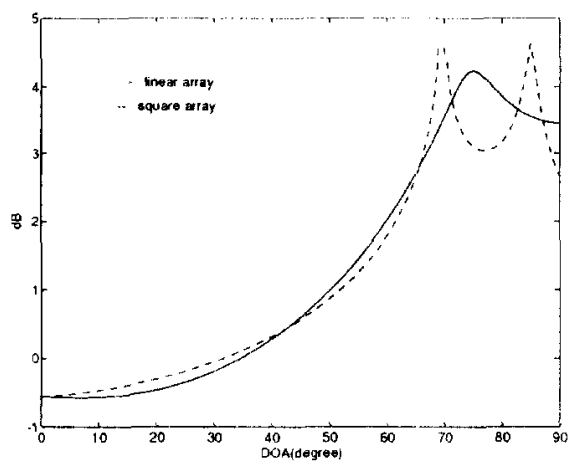

Figure 2. S.S and MUSIC for DOA estimat on of two coherent signals at $70^{\circ}$ and $85^{\circ}$

In the second example, we use the same square array to receive two coherent signals at $40^{\circ}$ and $50^{\circ}$. The SNR is $20 \mathrm{~dB}$. We applied $S . S$. and FBSS method individually.
Figure 3. shows that the DOA estimation resolution are much improved by using FBSS method.

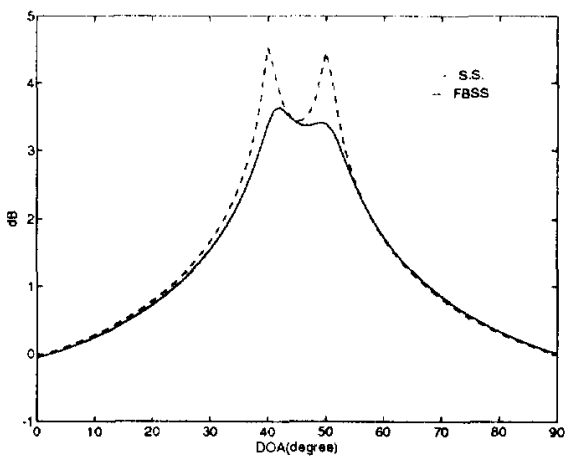

Figure 3. DOA estimation of two coherent signals at $40^{\circ}$ and $50^{\circ}$ by using a nine sensor square array

In the third example, we use a dense square array with sixty four sensors. The array contains 4 subarrays each with 49 sensors. The spacing between two neighboring seusors is $0.45 \lambda$. Four coherent signals at $20^{\circ}, 65^{\circ}, 150^{\circ}$ and $200^{\circ}$, and three other coherent signals at $230^{\circ}, 250^{\circ}$ and $280^{\circ}$, two other coherent signals at $30^{\circ}$ and $300^{\circ}$ and the other at $320^{\circ}$ are received by the array. The SNR is $20 \mathrm{~dB}$. 500 data samples were used to estimate the array output covariance matrix. First, we applied the FBSS and then applied MUSIC. Simulation results are shown in Fig.4.

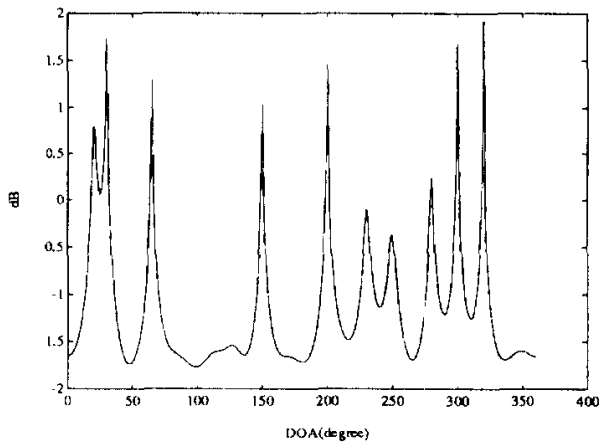

Figure 4. DOA estimation of four groups of coherent signals at $\left(20^{\circ}, 65^{\circ}, 150^{\circ}, 200^{\circ}\right),\left(230,250^{\circ}, 280^{\circ}\right) .\left(30^{\circ}, 300^{\circ}\right)$ and $320^{\circ}$ based on a sixty-four sensor square array

In the fourth example, we use the same array as in the third example to receive two coherent signals, one is at an azimuth of $40^{\circ}$ and an elevation of $30^{\circ}$, the other is at an 
azimuth of $50^{\circ}$ and an elevation of $60^{\circ}$. The SNR is $20 \mathrm{~dB}$. We applied FBSS method. Results is given in Fig. 5 .

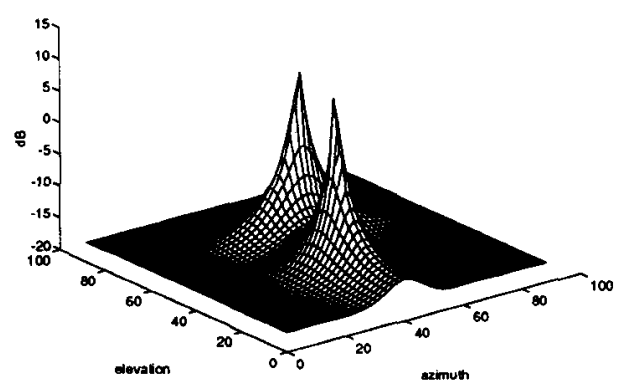

Figure 5. DOA estimation of two coherent signals at an azimuth of $40^{\circ}$ and an elevation of $30^{\circ}$, and at an azimuth of $50^{\circ}$ and an elevation of $60^{\circ}$, respectively

In the fifth example, we use a twelve-sensor array, consisting of two overlaped nine sensor square arrays as in example one, to receive two coherent signals at $70^{\circ}$ and $80^{\circ}$. The SNR is $20 \mathrm{~dB}$. 2000 trials were run. Histogram of the results is given in Fig.6. We applied FBSS first and then applied the ESPRIT. Two angles were clearly identified.

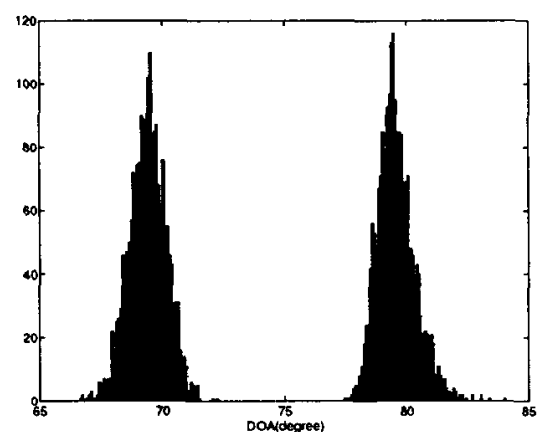

Figure 6. FBSS and ESPRIT for DOA estimation of two coherent signals at $70^{\circ}$ and $80^{\circ}$

\section{SUMMARY AND CONCLUSIONS}

To significantly improve robustness of DOA estimation, we developed techniques for applying S.S. on arrays with arbitrary geometry, thus making MUSIC and ESPRIT operative in a coherent interference environment. In order to apply S.S. to an array with arbitrary geometry, this array must have an orientational invariant structure and its center array must be ambiguity free. Also the number of subarrays must be greater than or equal to the largest number of mutually coherent signals. Such an array must also satisfy conditions for applying MUSIC. For ESPRIT, two identical arrays (or subarrays) separated by a displacement vector are used each satisfying the conditions for applying S.S. and MUSIC.

To get ambiguity free array manifolds for subarrays and center array, we need to ensure that no more than $\left\lceil\frac{K}{2}\right\rceil$ sensors are on one line and for any sensor in the array, there are at least another two sensors within the range of $\frac{\lambda}{2}$.

We evaluated FBSS on a nonlinear array with central symmetry and found that this technique outperform regular S.S. in terms of improved efficiency and estimation resolution. All the predicted results in this work are verified by simulations.

\section{REFERENCES}

[1] R. O. Schmidt, "A signal subspace approach to multiple source location and spectral estimation," Ph.D. dissertation, Stanford University, Stanford, CA, May 1981.

[2] R. Roy and T. Kailath, "ESPRIT-estimation of signal parameters via rotational invariance techniques," IEEE Trans. Acoust., Speech, Signal Processing, vol. 37 , No. 7 , July 1989 .

[3] J.E. Evans, J.R. Johnson and D.F. Sun, "High Resolution Angular Spectrum Estimation Techniques for Terrain Scattering Analysis and Angle of Arrival Estimation", in 1st ASSP Workshop Spectral Estim., Hamilton (CANADA), 1981.

[4] T. J. Shan, M. Wax and T. Kailath, "On spatial smoothing for Direction-of-Arrival estimation of coherent signals, "IEEE Trans. Acoust., Speech, Signal Processing, vol. 33, No. 4, pp.806-811, August 1985.

[5] S. U. Pillai and B. H. Kwon, "Forward/backward spatial smoothing techniques for coherent signal identifcation," IEEE Trans. Acoust., Speech, Signal Processing. vol. 37, No. 1, January 1989

[6] J. T.-H. Lo and S. L. Marple, "Observability conditions for multiple signal direction finding and array sensor localization," IEEE Trans. Signal Processing, vol. 40, No. 11, pp. 2641-2650, November 1992.

(7) $M$. Viberg, B. Ottersten and T. Kailath "Detection and estimation in sensor arrays using weighted sub space futing" IEEE Trans. Signal Processing vol. 39, No. 11, November 1991.

[8] J.A. Cadzow, "A high resolution direction-of-arrival algorithm for narrow-band coherent and incoherent sources," IEEE Trans. Acoust., Speech, Signal Processing, vol. 36, pp. 965-979, July 1988. 